\title{
De la parole à la carte : repérage, analyse et visualisation automatique de la perception d'une ville
}

\author{
Hélène Flamein ${ }^{1}$ et Iris Eshkol-Taravella ${ }^{2}$ \\ ${ }^{1}$ Laboratoire Ligérien de Linguistique, Université d'Orléans, UMR7270 \\ ${ }^{2}$ MoDyCo, Université Paris - Nanterre, UMR7114
}

\begin{abstract}
Résumé. A l'heure où de plus en plus de corpus et de données sont accessibles, le travail initié s'interroge sur l'exploitation de données linguistiques dans un corpus d'oral à dimension sociolinguistique avec l'objectif d'en extraire automatiquement du contenu subjectif. A partir de l'exploitation du corpus ESLO (Enquête Sociolinguistique à Orléans), l'objectif est de modéliser, détecter et visualiser la perception qu'ont les locuteurs de la ville d'Orléans grâce à une approche pluridisciplinaire. Cet article présente la chaîne de traitement développée pour atteindre cet objectif. A partir d'une première étape de détection des noms de lieux dans le corpus, une analyse de la polarité est réalisée. Les informations extraites sont projetées sous la forme d'une carte afin de matérialiser la perception des habitants de leur environnement.
\end{abstract}

\begin{abstract}
Oral transcript processing for automatic recognition, analysis and visualization of the perception of a city. At a time when an increasing number of corpora and data are accessible, the research done in this article aims the exploitation of linguistic data in an oral corpus with a sociolinguistic dimension in order to automatically extract subjective content. Based on the exploitation of the ESLO corpus (Sociolinguistic Survey in Orleans), the purpose is to model, detect and visualise the perception the speakers have of the city of Orleans through a multidisciplinary approach. This paper presents the processing chain developed to achieve this goal: the detection of the location names, the analysis of identified opinions and the visualisation of extracted information.. The extracted data is projected as a map in order to materialise the inhabitants' perception of their environment.
\end{abstract}

\section{Introduction}

A l'heure où le numérique est omniprésent, de plus en plus de corpus et de données sont accessibles. Articles de presse, productions littéraires, modes d'emploi et désormais commentaires d'utilisateurs sur le web, conversations par messageries instantanées, SMS, tweets ou encore contenus vidéoludiques ne sont que des exemples de la variété de données disponibles aujourd'hui. Le traitement de ces données multimodales renferme différents 
enjeux dont les principaux seraient de gérer la quantité incommensurable de données disponibles tout en étant capable de s'adapter à leur diversité de contenu et de forme. Pour répondre à ces enjeux, il est nécessaire d'avoir une approche pluridisciplinaire associant principalement la linguistique, le TAL et la géographie. Le travail présenté s'intéresse à la perception qu'ont les habitants de leur ville. Plus précisément, il s'agit d'extraire cette perception d'un corpus de conversations orales tiré d'ESLO (Enquête SocioLinguistique à Orléans). Ce type de données est à notre connaissance peu exploité dans les travaux existants en TAL et nous proposons de répondre à ce manque en présentant une méthodologie pour leur traitement et la visualisation de leur contenu afin d'en faciliter l'exploitation.

La chaîne de traitement conditionnant l'analyse de la perception se décompose en trois étapes : la détection des noms de lieux, l'analyse des opinions identifiées et la visualisation des informations extraites.

\section{Corpus et problématique}

\subsection{Enquête SocioLinguistique à Orléans}

Le corpus étudié est le corpus ESLO ${ }^{\mathrm{i}}$ (Enquête Sociolinguistique à Orléans) et, plus précisément le corpus ESLO2, la suite d'une enquête réalisée au début des années 70 par des chercheurs britanniques (ESLO1). Il comprend 18 modules représentant chacun une situation d'enregistrement différente : interviews d'habitants et de personnalités de la ville, des captations de paroles dans la rue, les transports publics, les commerces, les lieux de travail, etc. Le corpus ESLO est une ressource riche tant sur le plan qualitatif que quantitatif. L'ensemble du corpus est disponible en ligne et destiné aux communautés scientifiques intéressées par l'étude du français oral, ainsi qu'au grand public curieux d'en découvrir le contenu.

Deux modules favorisant la présence de noms de lieux ont été sélectionnés pour ce travail : Entretiens (84 transcriptions, 1167000 mots, discussions en face à face entre un enquêteur et un locuteur témoin à partir d'une trame d'entretien semi-directive) et Itinéraire (91 transcriptions, 70000 mots, enregistrements plus courts en micro-trottoir dans lesquels les enquêteurs demandent leur chemin au locuteur témoin).

\subsection{Difficultés de l'oral}

Le langage oral diffère de celui de l'écrit. L'une des caractéristiques principales de l'oral est la présence de disfluences (Blanche-Benveniste et al. 1990 ; Riegel et al. 1994). Les disfluences sont les « marques typiques des énoncés en cours d'élaboration » (Dister et al., 2009). Ces éléments rompent le flux de parole de façon aléatoire et révèlent les traces de construction du discours en temps réel (Blanche-Benveniste et al. 1990). Parmi les disfluences en français, on peut retrouver des marqueurs discursifs (quoi, enfin, ...), des onomatopées ou interjections (ah, euh, hum, ...), des répétitions, des reformulations, ou encore des amorces de mots.

y a quoi par-là y a le cons- euh une partie du conservatoire y a oui si c'est Sainte-Croix quoi la cathédrale. (ESLO2_iti_10_04)

Dans l'exemple a), l'expression cons- est l'amorce du lieu conservatoire. A cet instant, le locuteur hésite comme en témoigne l'onomatopée euh, puis, il se reprend pour donner la forme complète une partie du conservatoire. Par ailleurs, le marqueur discursif quoi apparaît au milieu du nom de lieu Sainte-Croix la cathédrale. Au-delà de la présence des disfluences, l'instantanéité du discours oral a une influence sur la façon dont un locuteur 
mentionne un lieu. Dans l'exemple 14, le lieu la cathédrale Sainte-Croix est mentionné par le locuteur dans l'ordre inverse Sainte-Croix la cathédrale ce qui ne se produira pratiquement jamais dans le discours écrit. A l'oral, il est extrêmement rare que quelqu'un fasse allusion à la cathédrale en utilisant son nom complet. Son nom officiel est : Cathédrale Sainte-Croix. Dans l'exemple a), le locuteur utilise les éléments du nom complet mais les donne dans le désordre. Il y fait allusion d'abord par le nom propre, et précise ensuite le type du monument.

Le traitement de l'oral diffère de celui de l'écrit et pose certaines difficultés. En chaque maillon de la chaîne de traitement, les spécificités propres de l'oral sont analysées et prises en compte dans l'élaboration des différents modules d'annotation et d'extraction.

\section{Détection des lieux}

\subsection{Notion de lieux}

La notion de lieu est une notion complexe. Sa définition pose question que ce soit du point de vue de la géogrpahie, de la linguistique, et du TAL. Dans leur Introduction à la Géographie Humaine, Bailly \& Béguin (2005) évoquent la difficulté de poser une définition précise du terme et propose de le considérer comme un point dans l'espace que l'on peut situer sur une carte indépendamment des relations qu'il peut entretenir avec d'autres lieux. C'est un endroit où l'on peut situer des phénomènes géographiques. Diverses nuances existent mais, au delà de l'analyse spatiale pure, ces discussions introduisent toute l'idée de l'existence d'une relation entre un ou des individus avec une portion de l'espace, ou dans une portion de l'espace (Tuan, 1977). C'est l'homme qui, par son histoire, son vécu, effectue la dénomination de l'espace qui l'entoure.

Du point de vue de la linguistique, la notion du lieu en français est majoritairement étudiée à travers l'étude des expressions spatiales (Boons, 1987; Borillo, 1998 ; Laur, 1991 ; Vandeloise, 1986 ; Le Pesant, 2011, 2012 ; etc.). La toponymie est une branche de l'onomastique qui s'intéresse à l'histoire, l'évolution, la signification des noms de lieux par rapport à une langue ou une communauté donnée. Bouvier (1999) définit deux classes de toponymes. Les toponymes de création relève de la décision d'un pouvoir généralement municipal qui impose l'usage d'un nom à tous les utilisateurs d'un lieu (rue de la Paix, Paris, Manhattan, etc.). Les toponymes d'usage quant à eux résultent d'un accord implicite entre les membres d'une communauté sur la manière de désigner un référent qui leur est commun (rue de la gare, place du Marché, Paname, etc.). Les pratiques du lieu, les habitudes qui y sont liées ou une caractéristique propre à l'endroit ou même l'action du temps, peuvent inciter les usagers du lieu à employer un nom différent du toponyme d'origine pour s'y référer. Le nom d'un lieu n'est pas une simple étiquette sur un espace : il symbolise son histoire, les enjeux qui lui sont propres (Le Squère, 2006).

Les chercheurs du domaine du TAL abordent principalement les lieux du point de vue de leur détection automatique dans le cadre de la tâche de reconnaissance des entités nommées (REN). Les entités nommées sont des objets informationnels pertinents jouant le rôle de descripteurs de faits, d'événements, etc. (Nouvel et al., 2015, 2013). Selon Ehrmann (2008) les entités nommées représentent «toute expression linguistique qui réfère à une entité unique du modèle de manière autonome dans le corpus ». Ces entités représentent des objets textuels porteurs de sens généralement classés selon plusieurs catégories : lieux, personnes, organisations, dates, unités monétaires et pourcentages (Maurel et al., 2011 ; Nadeau \& Sekine, 2009 ; Chinchor, 1998). De nombreux outils de détection automatique sont dédiés à la reconnaissance des entités nommées (REN) en anglais comme le module Stanford NER (Finkel et al., 2005) ou le POLYGLOT NER présenté dans Al-Rafou et al. (2015). Pour le français, nous pouvons citer le module CasEN ${ }^{\mathrm{ii}}$ développé pour le logiciel 
Unitex iii (Paumier, 2009). Lesbeguerries (2007) s'intéresse aussi à la notion de lieu dans le cadre de la Recherche d'Information (RI). Il propose le terme entité spatiale qui recouvre la définition du lieu et rend explicite la dimension géolocalisable d'un lieu. La majorité des travaux dédiés à l'extraction automatique des entités spatiales reposent sur des données textuelles comme des récits de voyages (Loustau et. al, 2008), des œuvres littéraires (Moncla et al., 2016) ou des documents textuels (journaux, cartes géographiques anciennes, lithographies, cartes postale, ...) (Lesbeguerries, 2007)

\subsection{Difficultés de la détection automatique des lieux}

D'une manière générale, la tâche de REN présente certaines difficultés. L'un des problèmes principaux réside dans la polysémie de certaines entités, dont les lieux font partie. Un exemple bien connu est celui du terme orange : désigne-t-il la couleur, le fruit, la ville du Sud de la France ou la compagnie de téléphonie française ? La présence ou l'absence d'une majuscule, ou le contexte d'emploi (aller à Orange, souscrire chez Orange, manger une orange, la chemise orange) peuvent aider à faire la différence entre ces sens mais l'ambiguïté persiste souvent (J'adore Orange! Les oranges oranges). Parfois, les noms de lieux sont employés en tant que personne. On observe cet emploi dans les articles journalistiques : La Maison Blanche a déclaré que..., Paris lance un appel à Washington, etc. Dans ces cas-là, le contexte apporte de l'ambiguïté dans la tâche de typage des entités nommées.

Une variation dans le nommage des lieux est une difficulté supplémentaire. Que ce soit à l'oral ou à l'écrit, la nature morphologique des mots désignant un lieu peut varier. Les noms de lieux peuvent être composés d'un ou de plusieurs mots (place du Général de Gaulle, université d'Orléans, etc.), être liés ou non par un trait d'union (le quartier Orléans-La Source ou Orléans La Source, Saint-Jean-de-la-Ruelle, etc.), etc. Le nommage des lieux répond à certaines conventions définies arbitrairement, ce qui n'empêche pas pour autant les locuteurs de faire varier cette norme. Ainsi, on peut indifféremment employer les noms place de l'Arc de Triomphe ou la place de l'Étoile pour se référer à la place Charles De Gaulle à Paris. Il n'existe qu'une seule place de ce genre donc cela reste possible pour un système automatisé de déterminer qu'il s'agit du même endroit et d'attribuer les coordonnées géographiques correspondantes. Cependant, à quelle rue fait-on référence si l'on évoque la rue de l'église ou la rue de la gare lorsque l'on se trouve dans une ville disposant de plusieurs églises ou gares? Dans ces cas-là, si le contexte n'est pas suffisamment explicite, il devient très complexe, voire impossible dans certains cas de relier ces expressions à un emplacement exact.

Différentes opérations concourent à faire varier le nom du lieu de la norme. En plus de la substitution du nom du lieu par un autre, noms de lieux peuvent aussi être abrégés. C'est le cas notamment des noms de villes composés de plusieurs mots comme Aix-en-Provence qui devient Aix ou La Ferté-Saint-Aubin qui devient La Ferté dans l'exemple b). La troncation est un autre phénomène qui peut survenir comme dans l'exemple c) où la rue de République devient rue de la Rép-.

je passais pas La Ferté ça faisait loin hein ça me faisait cinquante kilomètres (ESLO2_ENT_1023)

tu vas tout au bout jusqu'à la rue de la Rép- tu vois où elle est?

(c) (ESLO2_iti_06_11)

Matérialiser le portrait de la ville d'Orléans en géolocalisant les lieux identifiés dans le corpus ESLO suppose de résoudre cette problématique de variation des noms de lieux. Que 
la mention de lieu soit conventionnelle ou non, il faut dans tous les cas pouvoir associer ces mentions avec les coordonnées géographiques correspondantes.

\subsection{Modélisation et annotation des lieux}

L'annotation automatique des lieux dans l'oral transcrit se fait en plusieurs étapes prenant chacune en compte les problèmes soulevés par l'extraction des lieux à l'oral.

Avant de procéder au traitement des données, une étape d'observation manuelle du corpus a permis de modéliser des lieux et d'établir des conventions d'annotation. Son élaboration s'appuie sur l'exploration manuelle d'un échantillon du corpus général et l'analyse de conventions existantes comme celles établies lors des campagnes d'évaluation ESTER 1 (2005) et $2(2009)^{\mathrm{iv}}$ ou ETAPE $(2012)^{\mathrm{v}}$. Afin de préciser la nature des lieux identifiés, trois informations sont requises :

- La typologie des lieux qui considère les villes, régions, pays, espaces supranationaux, les voies de circulation, les lieux physiques naturels, les monuments, les administrations, les commerces, les lieux à fonction noncommerciale ainsi que les lieux avec une fonction éducative.

- La zone géographique afin de distinguer les lieux situés à Orléans (zone 2), dans son agglomération (zone 1) ou en dehors (zone 0 ).

- Le label officiel qui correspond au nom officiel du lieu, sans variation.

Dans l'exemple (d), la mairie d'Orléans est donc considérée comme un lieu administratif (admin), situé à Orléans (zone 2) et dont le nom officiel est mairie d'Orléans.

$$
\begin{gathered}
\text { la }<\text { loc type }=\text { "admin" zone }=" 2 " \text { label }=\text { "mairie d'Orléans" }> \\
\text { mairie d'Orléans }</ \text { loc }>\text { elle est belle (ESLO_iti_02_03) }
\end{gathered}
$$

Un corpus de référence a été constitué manuellement en accord avec la convention établie à partir d'un nouvel échantillon du corpus général composé de quinze transcriptions. 2262 mentions de lieux ont été annotées dont 46\% sont situés à Orléans. L'annotation a été réalisée par deux annotatrices et un accord inter-annotateur a été calculé. Le calcul du Kappa de Cohen (Cohen, 1960) pour la comparaison des annotations de A1 et A2 est égal à 0,81 . Cet accord prend en compte l'ensemble des informations annotées manuellement (type, zone et label) ainsi que la délimitation dans le texte. Si l'on se rapporte à la grille de lecture proposée par Landis et Koch (1977), l'accord est considéré comme excellent.

Le processus de détection automatique de noms de lieux dans le corpus oral transcrit est décrit dans Flamein \& Eshkol-Taravella (2020). Nous présenterons ici une synthèse de ce travail.

La manipulation de conversations orales pose certaines difficultés dans le traitement informatique. Aussi, une étape de prétraitement est nécessaire pour parer ces difficultés. Cette étape consiste principalement en l'élimination des disfluences qui sont reconstituées à la fin du traitement.

D'une manière générale, le système utilise les méthodes symboliques et s'appuie sur l'exploitation de ressources lexicales Geonames ${ }^{\text {vi }}$, GEOFLA ${ }^{\text {vii }}$ et Data.gouv.fr ${ }^{\text {viii }}$. Ces bases de données référencient des noms de lieux normalisés, associés à des coordonnées géographiques permettant leurs géolocalisations sur une carte. En l'état, ces ressources ne suffisent pas pour l'annotation exhaustive des lieux puisqu'elles ne recensent que les noms officiels, conventionnels des lieux. Comme on peut le voir dans les exemples (b) et (c), les noms rue de la Rép- et La Ferté divergent de la norme. Ces formes ne seront donc pas référencées dans les lexiques exploités contrairement à leurs formes originelles rue de la République et La Ferté Saint-Aubin. C'est la raison pour laquelle ces ressources lexicales sont enrichies en variantes de noms de lieux. 
Ainsi, nous avons observé des récurrences dans la manière d'abréger les noms de voies où le locuteur ne conserve que le dernier mot (rue Ganguin au lieu de la rue Paul Gauguin, ou rue Madeleine au lieu de rue Porte Madeleine) ou groupe prépositionnel (rue de Sonis au lieu de la rue du Général de Sonis) composant le nom officiel ainsi que le mot caractérisant le type de voie. Dans le cas des noms de villes abrégés comme La Ferté, ce sont les derniers termes ou groupes prépositionnels qui peuvent être supprimés. Il en est de même pour des noms comme Rio pour Rio de Janeiro, Aix, pour Aix-en-Provence, Sully, ou encore Saint-Cyr pour Saint-Cyr-en-Val. A partir de ces différentes constatations, des abréviations de noms de voies et de villes sont générées et ajoutées aux ressources lexicales.

Des traitements spécifiques sont aussi réalisés pour traiter les formes tronquées comme rue de la Rép-. Dans les conventions de transcription du corpus ESLO, les mots non finalisés sont marqués par un tiret. Grâce à cet indice, le système établit une fenêtre d'observation et tronque artificiellement les ressources lexicales. Les noms de voies tronqués peuvent alors être appliqués sur la fenêtre d'observation. Dans l'exemple (c), le système peut ainsi faire le lien entre la séquence dans la fenêtre d'observation rue de la Rép- et sa forme d'origine rue de la République. Il annote donc le tour de parole de la manière suivante :

tu vas tout au bout jusqu'à la $<$ loc type="voie" zone="0" label="Rue de la

République">rue de la République $</$ loc $>$ tu vois où elle est ?

$$
\text { (ESLO2_iti_06_11) }
$$

Au-delà de l'intérêt de découvrir de nouvelles mentions dans le corpus, l'enjeu principal autour de la détection des noms de lieux tronqués et abrégés est de pouvoir relier la forme variante avec sa forme normalisée. Faire ce lien est primordial pour permettre l'élaboration de la carte finale présentant la perception de la ville d'Orléans.

D'autres désignations de lieux comme les hyperonymes ou les noms inventés (rue de la gare, boulangerie du coin, etc.) sont détectées en utilisant des règles et des patrons décrivant leur structure et les contextes de leur apparition.

Finalement, le module est évalué en termes de mesures de Rappel, Précision et FMesure à partir du corpus de référence pour valider l'efficacité du système. Le corpus de référence est composé de quinze transcriptions, référençant 2292 noms de lieux. L'évaluation du module prend en compte l'ensemble des annotations réalisées automatiquement : type de lieu, zone géographique, label officiel et délimitation de l'entité identifiée dans le texte. Le module obtient un Rappel de 0,90, une Précision de 0,93 et une F-Mesure de 0,91. Cette évaluation prend en compte l'efficacité du système pour reconnaître les frontières du nom du lieu et prouve des performances satisfaisantes dans la tâche de détection des désignations de lieux dans l'oral transcrit.

L'annotation des mentions de lieux dans le corpus sert d'ancrage pour la deuxième étape du traitement. A partir des mentions identifiées, une fenêtre d'observation est ouverte pour réaliser une analyse d'opinion, effectuée par apprentissage automatique.

\section{Détection de la perception}

\subsection{Notion de perception}

La perception est une notion vaste que la linguistique, la psychologie et même les sciences de l'information ont tenté de circonscrire. Le dictionnaire Larousse en ligne ${ }^{\mathrm{ix}}$ ainsi que le $T L F i^{x}$, définissent la perception comme l'action de « percevoir par les organes des sens » ou comme une « idée, compréhension plus ou moins nette de quelque chose ». Ces définitions 
démontrent que la perception est le résultat de la mise en relation d'un sujet avec un objet : depuis le processus de collecte d'information par le biais des sens jusqu'à la compréhension de l'objet qui en résulte. Selon Neisser (1967), lorsque nous percevons un objet, ses propriétés ne se révèlent pas à nous immédiatement : l'information est traitée, mémorisée et forme une représentation qui pourra être retrouvée et réutilisée. Les sens permettent d'observer la forme, la couleur, l'odeur ou encore le goût que peut avoir un objet. Pour donner une cohérence à cette liste de caractéristiques, des processus cognitifs interviennent nécessairement dans la structuration et l'interprétation des informations.

Du point de vue du TAL, la perception est le plus souvent abordée sous l'angle de l'analyse de sentiment (sentiment analysis) et de la fouille d'opinion (opinion mining). Plusieurs termes sont employés dans ces travaux de façon parfois ambiguë pour désigner des concepts subjectifs : opinion, sentiment, émotion, etc. (Harb et al. 2008 ; Pak \& Paroubek, 2010 ; Marchand, 2015 ; Sindhu \& Vadivu, 2019). Les cadres de cet article ne nous permettent pas de développer toutes ces notions. Les méthodes employées pour leur détection sont multiples. Les approches symboliques (Maurel et. al, 2007 ; Zhang, 2015 ; Ivanovna, 2019) s'appuient le plus souvent sur des dictionnaires de mots polarisés comme le General Inquirer ${ }^{x i}$, le lexique de l'Opinion Finder System ${ }^{\mathrm{xii}}$, le SentiWordNet ${ }^{\mathrm{xiii}}$, ou pour le français : le NRC Emotion Lexicon (Mohammad \& Turney, 2010, 2013) et le French Expanded Emotion Lexicon (FEEL) ${ }^{\mathrm{xiv}}$ (Abdaoui et al. 2017). Dans ces dictionnaires, une polarité (positive ou négative) ou même des émotions sont associées à chacune des entrées. Lorsque ces dictionnaires sont inclus dans une chaîne de traitement, un score de subjectivité peut être attribué au document analysé en comptabilisant les mots porteurs d'opinion, de sentiment, d'émotion, etc. ou de polarité.

Actuellement ce sont les techniques d'apprentissage automatique qui sont exploitées majoritairement pour détecter des avis etc., leurs cibles et pour y associer une polarité. (Pak \& Paroubek, 2010 ; Blake et al., 2010 ; Eichstaedt et al., 2015 ; Kumar \& Bala, 2016 ; Sindhu \& Vadivu, 2019). Les travaux actuels en TAL se fondent majoritairement sur les données issues du Web et s'intéressent dans une moindre mesure aux avis exprimés à l'oral. Les données permettant ce genre d'analyse sont certes moins facilement disponibles mais présentent tout autant d'enjeux pour la problématique de la fouille d'opinion et de l'analyse de sentiments.

Dans cet article, nous préférons le terme de perception considérée comme le processus de traitement d'informations recueillies à l'aide de récepteurs sensoriels dans le but de créer des représentations et des connaissances à propos de l'objet perçu. Percevoir un objet passe d'abord par son expérimentation physique et sensorielle. L'analyse immédiate de ces impressions prépare la construction d'une opinion à propos de l'objet. La perception est une expérience interne qui varie d'un individu à l'autre et qui peut être captée lorsque l'individu décide de la partager. C'est cet instant de partage de la perception qu'il s'agit de détecter pour dresser le portrait de la ville d'Orléans.

La méthodologie présentée propose de partir des méthodes classiques de détection de la subjectivité et de la polarité dans des données de nature différente, afin de proposer une nouvelle façon d'analyser la perception dans la perspective d'un traitement automatisé plus fin.

\subsection{Modélisation et annotation de la perception}

L'analyse automatique de la perception vise la détection des énoncés subjectifs et leur classification en fonction de la polarité selon plusieurs étapes. Tout d'abord, des conventions d'annotations sont établies. L'objectif de cette typologie est de caractériser la subjectivité et la polarité dans le corpus est présentée dans la figure 2.

Dans un premier temps, les énoncés objectifs sont distingués des énoncés subjectifs. Ensuite, la nature de la subjectivité est précisée au regard de la polarité. Chaque énoncé 
jugé subjectif est annoté en fonction de son orientation positive ou négative. Cette étude de la subjectivité et de la polarité des énoncés est la première étape vers l'analyse de la perception.

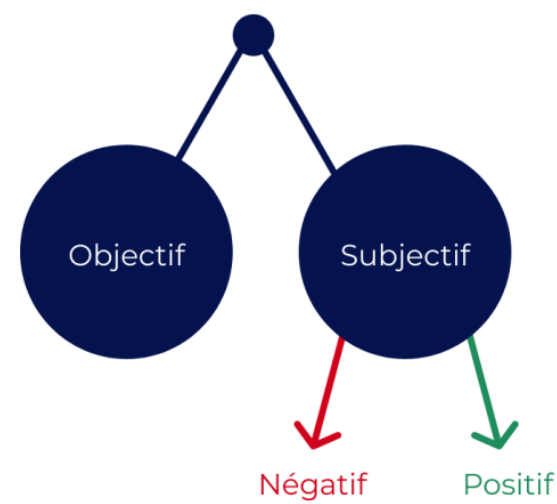

Fig. 2. Typologie de la subjectivité

Le corpus annoté manuellement est ensuite divisé en trois sous-corpus d'entraînement, de test et d'évaluation. Afin d'entraîner un modèle pour la détection de la subjectivité et de la polarité (D), différents features sont envisagés : la lemmatisation et l'étiquetage morphosyntaxique $^{\mathrm{xV}}$ des segments ainsi que le calcul d'un score de polarité et d'un score d'émotion $^{\mathrm{xvi}}$. Le TF-IDF et Word2Vec sont des méthodes possibles pour créer une représentation vectorielle des segments à analyser. Enfin deux classifieurs, RandomForest et SVM, ont été retenus pour réaliser la classification attendue. L'entraînement du modèle consiste à identifier quelle combinaison de features, de méthodes de vectorisation du texte et de classifieurs permet d'atteindre les objectifs de détection de la subjectivité et de la polarité dans le corpus.

Différentes expériences mêlant les différents features, les méthodes de représentations vectorielles et les classifieurs ont été menées pour sélectionner la combinaison la plus performante. Ainsi, un modèle d'apprentissage pour la détection de la subjectivité et la détection de la polarité a été établit. L'utilisation du classifieur SVM, la représentation vectorielle des segments lemmatisés avec le TF-IDF et l'utilisation de tous les features disponibles constitue le modèle le plus efficace. Les expériences révèlent que le même modèle est le plus efficace pour distinguer les énoncés subjectifs des énoncés objectifs mais aussi pour détecter la polarité des énoncés. Pour confirmer les performances de ce modèle, celui-ci est évalué sur les corpus de test définis. Les résultats de cette évaluation sont présentés dans le tableau 1. Pour la tâche de détection de la subjectivité, le modèle obtient donc une macro-average de 0,77 et une macro-average de 0,76 pour le traitement de la polarité.

Tableau 1. Extrait des expérimentations réalisées pour la détection de la subjectivité.

\begin{tabular}{|c|c|c|c|c|c|c|}
\hline Classifieur & Features & Cible & Macro average & Précision & Rappel & F-mesure \\
\hline \multirow{2}{*}{$\begin{array}{l}\text { TF-IDF } \\
+ \text { SVM }\end{array}$} & \multirow{2}{*}{$\begin{array}{c}\text { Score polarité } \\
+ \text { Score émotions } \\
+ \text { POS }\end{array}$} & subj. & \multirow{2}{*}{0,77} & 0,69 & 0,56 & 0,63 \\
\hline & & obj. & & 0,77 & 0,89 & 0,83 \\
\hline
\end{tabular}




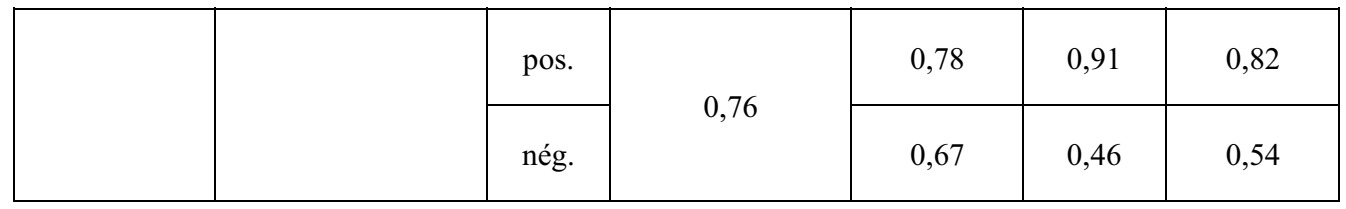

D'une manière générale nous remarquons que, moins il y a de traitements appliqués aux segments lors de leur vectorisation, meilleurs sont les résultats. En effet, les méthodes de vectorisation limitant le nombre de dimensions des vecteurs profitent à la qualité de la classification réalisée par SVM. La piste de la réduction de dimension des vecteurs doit être approfondie pour améliorer les performances du classifieur. Dans le même temps, l'association des scores de polarité et d'émotions et des POS participe à l'optimisation du modèle. Il semble aussi nécessaire d'équilibrer la composition du corpus de référence, en ajoutant en particulier des segments polarisés négativement. Une nouvelle phase d'annotation manuelle doit donc être réalisée. Enfin d'autres features, comme le nombre de mots composant les segments, la position des termes porteurs de polarité dans le segment, devront être identifiés pour améliorer l'apprentissage du modèle.

\section{Visualisation de la perception}

L'objectif de la dernière étape de ce travail est de représenter visuellement les résultats des analyses réalisées jusqu'ici. Cette étape commande de réfléchir aux moyens les plus adaptés pour permettre la visualisation de la perception. La question de la visualisation des informations est essentielle du point de vue de la géographie. L'élaboration de cartes ou la mise en place de Systèmes d'Information Géographique est un enjeu majeur pour la représentation de l'information spatiale. Ces problématiques s'intègrent dans une logique pluridisciplinaire en cherchant à mettre en évidence les liens qu'entretient l'Homme avec l'espace. Il s'agit donc de mettre en valeur les informations extraites de notre corpus afin de faciliter leur manipulation et les rendre accessibles. Dans une autre mesure, la visualisation des lieux et des annotations réalisées contribue faire émerger les relations qui unissent les différentes informations détectées.

La visualisation de la perception par les Orléanais de leur ville est réalisée avec la mise en place d'un Système d'Information Géographique (SIG) et la création de cartes. Pour cela, nous utilisons l'outil ArcGIS Online ${ }^{\text {xvii }}$, développé par la société Esri ${ }^{\text {xviii }}$ et disponible en ligne. Plusieurs couches d'informations sont projetées dans le système et permettent de figurer les éléments suivants :

- L'ensemble des lieux détectés en fonction de leur type

- La densité des mentions de lieux à Orléans

- Le nombre de mentions de villes

- La polarité associée aux lieux d'Orléans

L'affichage des informations s'appuie sur différents paramètres. Par exemple, lorsqu'une couche contient un grand nombre d'entités, l'affichage individuel de chaque entité sur la carte est souvent inutile. En l'état, on ne voit pas la différence entre les lieux qui n'ont été mentionnés qu'une seule fois et ceux qui l'ont été des dizaines de fois. Dans cette représentation, les entités se superposent, ce qui empêche de les distinguer clairement. Même lorsqu'elles ne se superposent pas, il est généralement difficile, voire impossible, d'extraire visuellement les informations significatives lorsque des centaines ou des milliers de points sont affichés en même temps. 


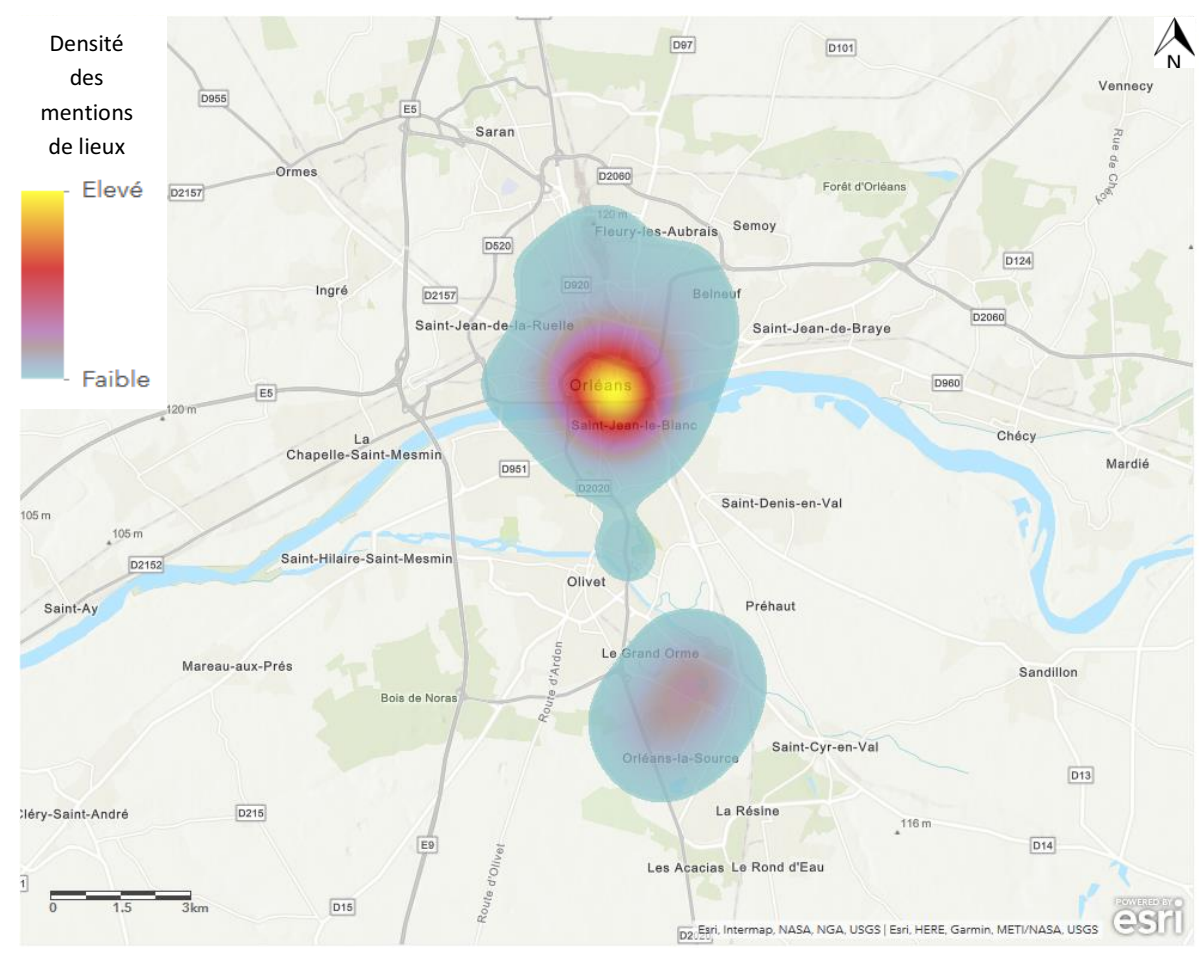

Fig. 5. Carte de densité des lieux d'Orléans

Pour résoudre ce problème, il est possible de générer une carte de densité (ou heat map). Une carte de densité représente la densité géographique des entités à représenter sur une carte à l'aide de zones colorées représentant ces points. Les zones les plus grandes sont celles où la concentration de points est la plus importante. La figure 5 illustre la carte de densité des lieux situés à Orléans : plus la couleur s'éloigne du bleu pour aller vers le jaune, plus la concentration de lieux identifiés est importante. On remarque que deux zones se distinguent. La première, et la plus dense, est située au niveau du centre-ville. C'est de ce quartier et des lieux qui s'y trouvent dont les locuteurs parlent le plus. La deuxième zone se situe au niveau du quartier Orléans La Source. Ces quartiers concentrent la plupart des services de la ville et il semble donc logique que ce soit de ces zones-là dont les gens parlent. La carte de densité révèle aussi un espace qui semble délaissé par les locuteurs, c'est-à-dire la partie sud du quartier Saint-Marceau. Aucun lieu n'a été ne serait-ce que mentionné dans cette partie du quartier. Nous avons établi que les locuteurs se référent aux espaces dans lesquels ils expérimentent, dont on leur parle. Ainsi, si cette partie de la ville n'est pas mentionnée, cela signifie que les locuteurs n'y vivent pas d'expériences particulières.

Dans une autre mesure, l'information du nombre d'occurrence d'un lieu peut aussi servir à accentuer d'autres paramètres. La figure 5 présente la polarité d'une partie des lieux situés à Orléans avec deux exemples de déclarations faites à propos de la cathédrale et de la place du Martroi. Un ratio compris entre 0 et 5 est calculé pour faire la part entre les déclarations positives et négatives. Dans cette couche, la taille du point accentue l'importance du ratio de polarité. Plus les occurrences sont nombreuses, plus le score obtenu aura du poids. Ainsi, on peut considérer la perception de la Loire comme réellement positive étant donné le grand nombre de déclarations portant à son sujet. Au contraire, la polarité sera à relativiser dans le cas des lieux mentionnés peu de fois. 


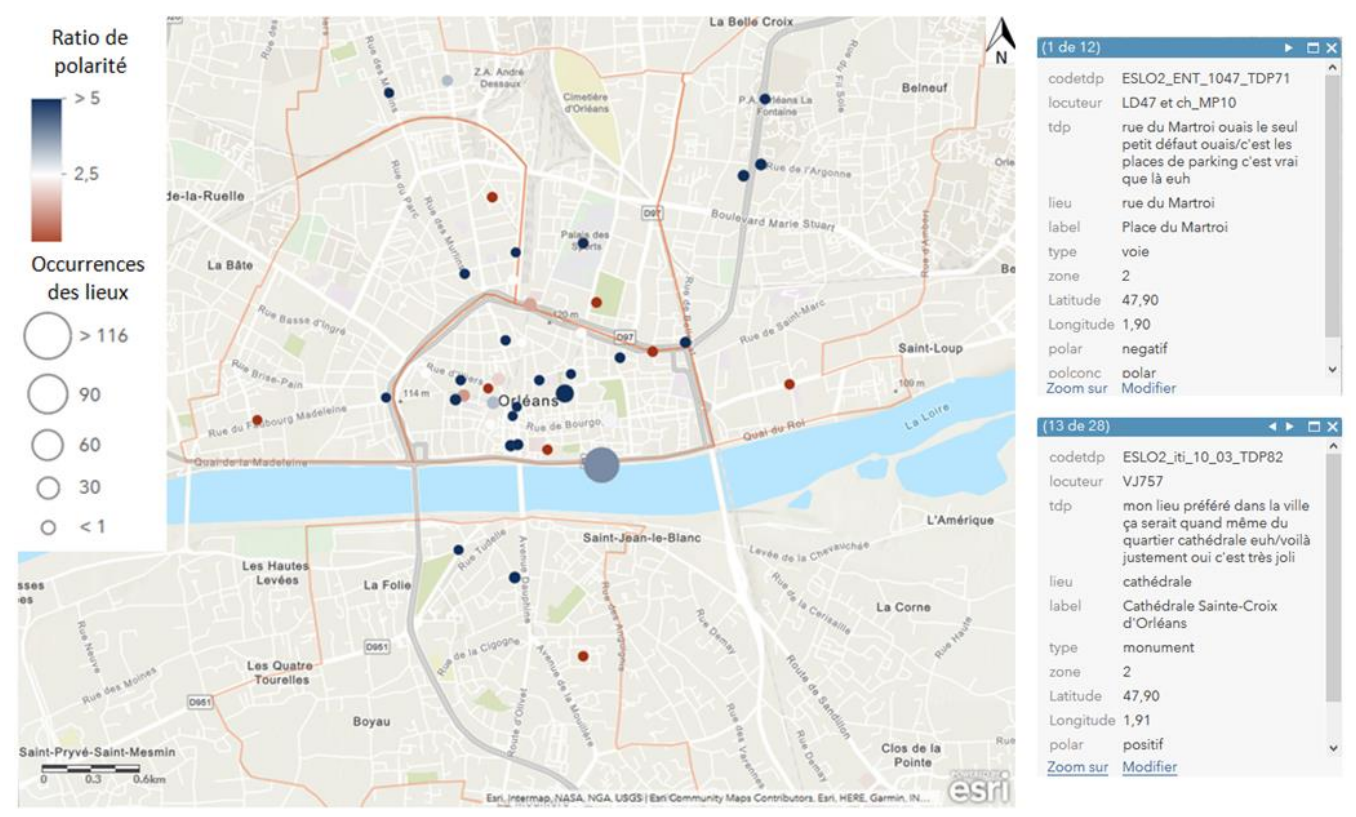

Fig. 5. Visualisation de la polarité des lieux d'Orléans dans le SIG

\section{Conclusions et perspectives}

A partir de l'exploitation du corpus de transcriptions d'enregistrements sonores ESLO, la finalité de cette étude est de modéliser, détecter et visualiser la perception des locuteurs de la ville d'Orléans. La démarche pluridisciplinaire présentée exploite les techniques variées du TAL (les méthodes symboliques et les méthodes d'apprentissage supervisé), les outils de la géographie (SIG) et les approches de la linguistique de corpus.

La détection de la subjectivité et de la polarité oriente l'analyse de la perception mais ne suffit pas à en rendre compte. Pour rendre compte de l'image qu'ils en ont, il est nécessaire de considérer d'autres informations que la polarité. Il faut approfondir la typologie de la perception pour en comprendre la nature et affiner son traitement automatique. La question est désormais de décrire sur quoi elle porte, à travers quoi ou de quelle façon elle s'exprime. A partir d'une exploration manuelle du corpus, nous proposons deux axes complémentaires selon lesquels on peut décrire la perception.

Dans un premier temps, nous proposons de nous intéresser à la cible même de la perception en décrivant les types d'objets mentionnés pour décrire la ville. Même si la trame de certains enregistrements guide la conversation et incite les locuteurs à parler de leurs lieux de vie et en général d'Orléans, les arguments pour le faire ne sont pas imposés. A l'instant où un locuteur est interrogé sur le sujet, il fait le choix, instantanément, d'évoquer certains éléments plutôt que d'autres. Le fait de mettre l'accent sur certaines thématiques pour décrire la ville est révélateur de la représentation que se fait la personne de son environnement et donc de sa perception de celle-ci. L'exploration manuelle du corpus révèle ainsi quatre thématiques : urbanisme, sociale, économique et historicoculturelle.

Dans un second temps, la perception peut aussi être vue à travers les habitudes des individus, des actions qu'ils réalisent, de leurs goûts ou de ce qu'ils expérimentent par les sens. Ces éléments décrivent la nature de la perception et recoupent les différentes thématiques pouvant être abordées. Pour décrire la nature de la perception, nous considérons trois catégories : 
- La perception sensorielle qui considère le positionnement d'un individu par rapport à son environnement via le prisme de ses sens

- L'expérience personnelle qui représente les habitudes prises ou les activités menées dans un lieu par l'individu qui en parle. Le locuteur choisi de mettre en avant des expériences dans lequel il est lui-même impliqué, ce qui en accentue l'importance.

- L'expérience collective qui correspond aux expériences et représentations relatives à la ville d'autres personnes par le locuteur.

Cette réflexion prospective donne un premier cadre pour une analyse approfondie de la perception d'une ville faites par ses habitants.

\section{Références bibliographiques}

Abdaoui, A., Azé, J., Bringay, S., and Poncelet, P. (2017). FEEL : A French Expanded Emotion Lexicon. Language Resources and Evaluation, 51(3), 833-855.

https://doi.org/10.1007/s10579-016-9364-5

Al-Rfou, R., Kulkarni, V., Perozzi, B., and Skiena, S. (2015). Polyglot-NER: Massive Multilingual Named Entity Recognition. Proceedings of the 2015 SIAM International Conference on Data Mining, Vancouver, British Columbia, Canada, April 30 - May 2, 2015.

Bailly, A., and Beguin, H. (2005). Introduction à la géographie humaine (éd. 8). Paris: Armand Colin.

Blake, B. P., Agarwal, N., Wigand, R. T., and Wood, J. D. (2010). Twitter Quo Vadis : Is Twitter bitter or are tweets sweet? 2010 Seventh International Conference on Information Technology: New Generations, 1257-1260. IEEE.

Blanche-Benveniste, C., Bilger, M., Rouget, C., Van Den Eynde, K., Mertens, P., and Willems, D. (1990). Le français parlé (études grammaticales). Sciences du langage.

Boons, J.-P. (1987). La notion sémantique de déplacement dans une classification syntaxique des verbes locatifs. Langue française, (76), 5-40.

Borillo, A. (1998). L'espace et son expression en français. Editions Ophrys.

Bouvier, J.-C. (1999). Odonymes d'agglomération entre l'écrit et l'oral. Nouvelle revue d'onomastique, 33(1), 303-310.

Chinchor, N. A. (1998). Overview of muc-7/met-2. Science Applications International Corp San Diego CA.

Clark, H. H. (1996). Using Language. Cambridge University Press.

Cohen, J. (1960). A coefficient of agreement for nominal scales. Educational and psychological measurement. Vol. 20, $\mathrm{n}^{\circ} 1$, pp. 37-46.

Cui, W., Wu, Y., Liu, S., Wei, F., Zhou, M. X., and Qu, H. (2010). Context preserving dynamic word cloud visualization. 2010 IEEE Pacific Visualization Symposium (PacificVis), 121-128. https://doi.org/10.1109/PACIFICVIS.2010.5429600

Dister, A., Constant, M., and Purnelle, G. (2009). Normalizing speech transcriptions for Natural Language Processing. 15.

Ehrmann, M., Nouvel, D., and Rosset, S. (2016). Named Entity Resources-Overview and Outlook. Portorož, Slovenia. 
Eichstaedt, J. C., Schwartz, H. A., Kern, M. L., Park, G., Labarthe, D. R., Merchant, R. M., Jha, S., Agrawal, M., Dziurzynski, L. A., Sap, M., Weeg, C., Larson, E. E., Ungar, L. H., Seligman M. E. P. (2015). Psychological language on Twitter predicts county-level heart disease mortality. Psychological science, 26(2), 159-169.

Finkel, J. R., Grenager, T., and Manning, C. (2005). Incorporating non-local information into information extraction systems by gibbs sampling. Proceedings of the 43rd annual meeting on association for computational linguistics, 363-370. Association for Computational Linguistics.

Flamein, H., and Eshkol-Taravella, I. (2020). Noms de lieux dans le corpus de français parlé : Une approche symbolique pour un traitement automatisé. Le français moderne, n. 1. Editions CILF.

Heimerl, F., Lohmann, S., Lange, S., and Ertl, T. (2014). Word Cloud Explorer : Text Analytics Based on Word Clouds. 2014 47th Hawaii International Conference on System Sciences, 1833-1842. https://doi.org/10.1109/HICSS.2014.231

Novakova, I. (2019). Le lexique des émotions. UGA Éditions.

Kergosien, E., Laval, B., Roche, M., and Teisseire, M. (2014). Are opinions expressed in land-use planning documents? International Journal of Geographical Information Science, 28(4), 739-762.

Kergosien, E., Maurel, P., Roche, M., and Teisseire, M. (2013). OPITER: Fouille de données d'opinion pour les territoires. Spatial Analysis and GEOmatics (Sagéo'13), Brest.

Kergosien, E., Maurel, P., Roche, M., and Teisseire, M. (2015). Senterritoire pour la détection d'opinions liées à l'aménagement d'un territoire. Revue Internationale de Géomatique, 25(1), 11-34. https://doi.org/10.3166/RIG.25.11-34

Kumar, M., and Bala, A. (2016). Analyzing Twitter sentiments through big data. 2016. 3rd International Conference on Computing for Sustainable Global Development (INDIACom), 2628-2631. IEEE.

Landis, J. R. and Koch, G. G. (1977). The measurement of observer agreement for categorical data. biometrics. pp. 159-174.

Laur, D. (1991). Sémantique du déplacement et de la localisation en français : Une étude des verbes, des prépositions et de leurs relations dans la phrase simple ( $\mathrm{PhD}$ Thesis). Toulouse 2.

Le Pesant, D. (2011). Problèmes de morphologie, de syntaxe et de classification sémantique dans le domaine des prépositions locatives.

Le Pesant, D. (2012). Essai de classification des prépositions de localisation. SHS Web of Conferences, 1, 921-937. EDP Sciences.

Le Squère, R. (2006). Analyse des perceptions, usages et fonctions des toponymes actuels des territoires ruraux et urbains de Bretagne. Cahiers de sociolinguistique, (1), 81-99.

Lesbegueries, J., Sallaberry, C., and Gaio, M. (2006). Associating spatial patterns to textunits for summarizing geographic information. In ACM (Éd.), ACM SIGIR 2006. GIR, Geographic Information Retrieval, Workshop (p. 40-43).

Liu, B., and Zhang, L. (2012). A Survey of Opinion Mining and Sentiment Analysis. In C. C. Aggarwal and C. Zhai (Éd.), Mining Text Data (p. 415-463). https://doi.org/10.1007/9781-4614-3223-4_13 
Loustau, P., Nodenot, T., and Gaio, M. (2008). Spatial Decision Support in the Pedagogical Area : Processing Travel Stories to Discover Itineraries Hidden Beneath the Surface. In L. Bernard, A. Friis-Christensen, and H. Pundt (Éd.), The European Information Society: Taking Geoinformation Science One Step Further (p. 359-378). https://doi.org/10.1007/978-3-540-78946-8_19

Marchand, M. (2015). Domaines et fouille d'opinion: une étude des marqueurs multipolaires au niveau du texte. PhD Thesis.

Maurel, D., Friburger, N., Antoine, J. Y., Eshkol-Taravella, I., and Nouvel, D. (2011). CasEN: a transducer cascade to recognize French Named Entities. TAL, 52(1), 69-96.

Maurel S., Curtoni P. and Dini L. (2007). Classification d'opinions par méthodes symbolique, statistique et hybride. Actes de DEFT 2007, p. 111-117, [en ligne] [URL : http://www.celi-france.com/IMG/pdf/celi-france_deft07-2.pdf]

Mohammad, S. M., and Turney, P. D. (2010). Emotions evoked by common words and phrases : Using mechanical turk to create an emotion lexicon. Proceedings of the NAACL HLT 2010 workshop on computational approaches to analysis and generation of emotion in text, 26-34. Association for Computational Linguistics.

Mohammad, S. M., and Turney, P. D. (2013). Crowdsourcing a Word-Emotion Association Lexicon. Computational Intelligence, 29(3), 436-465. https://doi.org/10.1111/j.14678640.2012.00460.x

Moncla, L., Gaio, M., Nogueras-Iso, J., and Mustière, S. (2016). Reconstruction of itineraries from annotated text with an informed spanning tree algorithm. International Journal of Geographical Information Science, 30(6), 1137-1160. https://doi.org/10.1080/13658816.2015.1108422

Nadeau, D., and Sekine, S. (2009). A survey of entity recognition and classification. Named entities-recognition, classification and use. Amsterdam/Philadelphia: John Benjamins Publishing Company.

Neisser, U. (1967). Cognitive psychology appleton-century-crofts. New York, 351.

Nouvel, D., Ehrmann, M., and Rosset, S. (2016). Named Entities for Computational Linguistics. ISTE.

Nouvel, D., Antoine, J.-Y., Friburger, N., and Soulet, A. (2012). Coupling Knowledge-based and Data-driven Systems for Named Entity Recognition. Proceedings of the Workshop on Innovative Hybrid Approaches to the Processing of Textual Data, 69-77.

Pak, A., and Paroubek, P. (2010). Twitter as a corpus for sentiment analysis and opinion mining. LREc, 10, 1320-1326.

Paumier, S., Nakamura, T., and Voyatzi, S. (2009). UNITEX, a Corpus Processing System with Multi-Lingual Linguistic Resources. eLEX2009, 173.

Shriberg, E. E. (s. d.). Preliminaries to a Theory of Speech Disfluencies. 225.

Sindhu, C., and Vadivu, G. (2019). Sentiment Analysis and Opinion Summarization of Product Feedback. International Journal of Recent Technology and Engineering (IJRTE), 8 .

Tuan, Y.-F. (1977). Space and place: The perspective of experience. U of Minnesota Press.

Vandeloise, C. (1986). L'espace en français : Sémantique des prépositions spatiales (Vol. 13). Seuil. 
Zhang, L. (2012). Analyse automatique d'opinion: Problématique de l'intensité et de la négation pour l'application à un corpus journalistique (Université de Caen).

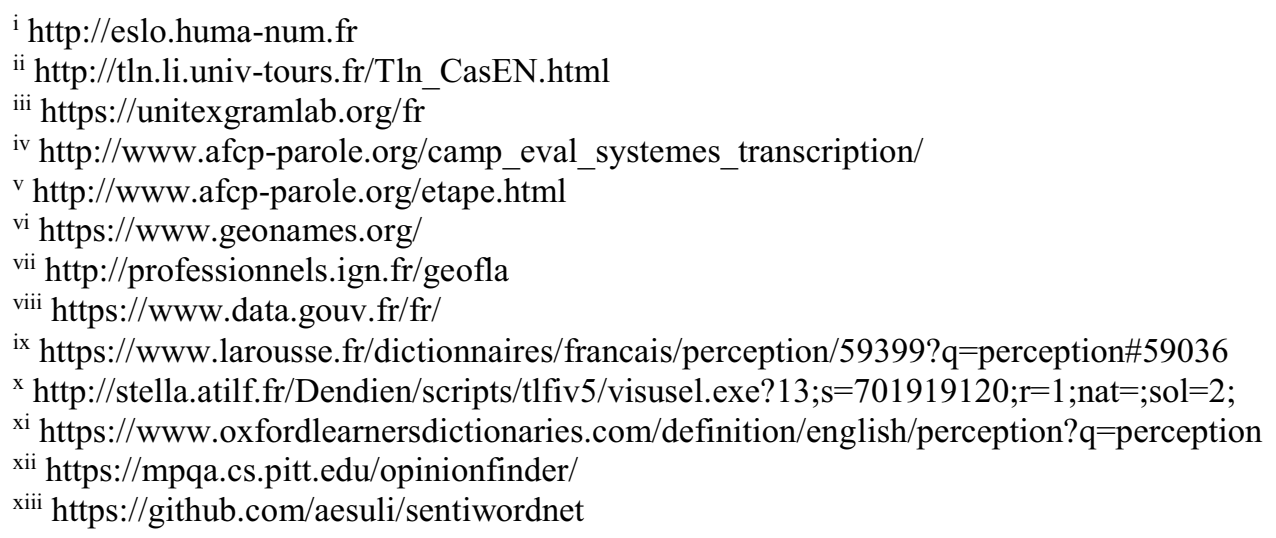

${ }^{\mathrm{xv}}$ Réalisé grâce à Treetaggerwrapper : https://treetaggerwrapper.readthedocs.io/en/latest/ xvi Réalisé grâce à PyFeel :https://github.com/AdilZouitine/pyFeel

xvii https://www.arcgis.com/index.html

xviii https://www.esri.com/fr-fr/home 